\title{
The origin of photoluminescence from thin films of silicon-rich silica
}

\author{
A. J. Kenyon, ${ }^{\text {a) }}$ P. F. Trwoga, and C. W. Pitt \\ Department of Electronic and Electrical Engineering, University College London, Torrington Place, \\ London, WC1E 7JE \\ G. Rehm \\ Friedrich-Alexander Universitat, Erlangen, Nürnberg, Germany
}

(Received 22 January 1996; accepted for publication 11 March 1996)

\begin{abstract}
We have carried out a study of the photoluminescence properties of silicon-rich silica. A series of films grown using plasma enhanced chemical vapor deposition over a range of growth conditions were annealed under argon at selected temperatures. Photoluminescence spectra were measured for each film at room temperature and for selected films at cryogenic temperatures. The photoluminescence spectra exhibit two bands. Fourier transform infrared and electron spin resonance spectroscopies were used to investigate bonding and defect states within the films. The data obtained strongly suggest the presence of two luminescence mechanisms which exhibit different dependencies on film growth conditions and postprocessing. We make assignments of the two mechanisms as (1) defect luminescence associated with oxygen vacancies and (2) radiative recombination of electron-hole pairs confined within nanometer-size silicon clusters ("quantum confinement'). (C) 1996 American Institute of Physics. [S0021-8979(96)04912-2]
\end{abstract}

\section{INTRODUCTION}

Light emission from silicon-based devices is an important research area in the electronics industry, as the realization of such devices would make it possible to capitalize on the broad base of knowledge available and to efficiently interface with existing electronics technologies. However, the indirect band gap of silicon means that it is a very inefficient light source. This has so far precluded the achievement of true large scale optoelectronic integration at reasonable cost.

Recent developments of new forms of silicon have renewed interest in the production of silicon-based luminescent devices and there is currently significant activity in the field of light emission from novel forms of silicon. Following Canham's initial report of porous silicon ${ }^{1}$ there has been an explosion of interest into its novel optical properties, and indeed it does seem to generate light surprisingly efficiently for an indirect band-gap material. Figures quoted for quantum efficiency are typically around $5 \%{ }^{2}$ There are now a large number of groups working on many aspects of porous silicon, from fundamental theory ${ }^{3-5}$ to the production of prototype luminescent devices. ${ }^{6-8}$ However, the recognized instability of the material may suggest that it is perhaps less suitable for general device use than was originally thought. Reduction in light output after prolonged exposure to the atmosphere and light-induced degradation continue to pose problems. ${ }^{9,10}$

A related material which has recently begun to generate interest in silicon-rich silica $\left(\mathrm{SiO}_{x}, x<2\right)$, arguably a more robust material which exhibits similar optical properties to porous silicon but is significantly less susceptible to damage. It consists of nanometer-size silicon aggregates suspended in a silica matrix. Although luminescence was reported in this material some time ago, ${ }^{11}$ it is only recently that its optical properties have begun to be thoroughly studied. A number of

${ }^{a)}$ Electronic mail: t.kenyon@eleceng.ucl.ac.uk techniques have been employed in its production, including implantation of silicon into silica, ${ }^{12-14}$ implantation of oxygen into silicon, ${ }^{15,16}$ plasma enhanced chemical vapor deposition (PECVD) ${ }^{17,18}$ low-pressure chemical vapor deposition (LPCVD) ${ }^{19,20}$ and co-sputtering of silicon and oxygen. ${ }^{21,22}$ $\mathrm{X}$-ray diffraction and transmission electron microscopy (TEM) studies have confirmed the presence of clusters of silicon atoms in the $\mathrm{SiO}_{2}$ matrix. ${ }^{18}$ Depending on the deposition technique and postprocess annealing, these inclusions can be either amorphous or crystalline: initially amorphous clusters tend to crystallize on annealing. Recent results have demonstrated broadband light emission from the blue to near infrared from material containing silicon aggregates of varying sizes. ${ }^{12-18}$ However, there is considerable uncertainty about the nature of the luminescence mechanism from silicon-rich silica. A number of possibilities present themselves: radiative recombination of confined excitons within silicon clusters ("quantum dots"), ${ }^{23}$ defect luminescence, ${ }^{24}$ interfacial effects at cluster surfaces, ${ }^{25}$ and luminescence from novel siloxene molecules. ${ }^{26}$ Each of these proposed mechanisms has its proponents and supporting experimental evidence, leading to a very confused picture. Photoluminescence studies carried out to date have failed to conclusively identify a unique luminescence mechanism and there are a number of contradictory reports in the literature. Some groups have reported bright, broadband emission from asgrown thin films of silicon-rich silica, ${ }^{17}$ while other groups see nothing until their samples are annealed. ${ }^{27}$ While some reports show a redshift of peak luminescence wavelength with increasing annealing temperature, ${ }^{13,24,27}$ others exhibit a fixed spectral distribution. ${ }^{14,28}$ Similarly, it is not clear whether temperature quenching of luminescence is significant in this material: some groups show a strong dependence of luminescence intensity on sample temperature, ${ }^{28}$ others see no change on cooling samples to $70 \mathrm{~K}^{24}$

As yet there is no model which adequately explains the wide range of experimental observations: it seems possible 
that more than one mechanism contributes to luminescence. It may be that several mechanisms operate at once or that different processes are favored by different growth conditions and/or postprocessing. Recently, a few groups, notably Shimizu-Iwayama and co-workers, ${ }^{13,14}$ Fischer et al.,${ }^{17}$ and to some extent Komoda et al. ${ }^{12}$ have begun to make progress in identifying mechanisms. Studying silicon-implanted silica, Shimizu-Iwayama identified two bands in the photoluminescence spectrum, one around $2.2 \mathrm{eV}$ and another at $1.7 \mathrm{eV}$. Using electron spin resonance (ESR) spectroscopy, they detected the presence of a large number of defects in their samples prior to annealing. These defects are associated with oxygen vacancies in silica. ${ }^{13,14}$ The number of defect centers detected by ESR falls rapidly on annealing at temperatures in excess of $600{ }^{\circ} \mathrm{C}$, and the photoluminescence band appears to be strongly quenched at the same rate. Making a link between the two observations, Shimizu-Iwayama et al. proposed that defects are the source of the high-energy emission from $\mathrm{SiO}_{x}$, and some form of quantum confinement effect produces the $1.7 \mathrm{eV}$ band which does not appear until samples are annealed at temperatures above $1100^{\circ} \mathrm{C}$. In subsequent articles by the same group, the $2.2 \mathrm{eV}$ luminescence has been linked specifically with defects at the silicon cluster $/ \mathrm{SiO}_{2}$ interface. ${ }^{14}$ Komoda's results ${ }^{12}$ also indicated the presence of two bands in the luminescence spectrum of $\mathrm{Si}^{+}$implanted silica. There was evidence for both confinement of excitons and defect luminescence.

Studying silicon-rich silica films deposited using PECVD, Fischer et al. ${ }^{17}$ identified contributions to the photoluminescence from two distinct regions: small nanometersize silicon inclusions with relatively high carrier mobility, and highly localized luminescent centers within the bulk matrix. These conclusions were deduced from temperature quenching and temperature-related shifts in the energy of the photoluminescence band.

We have undertaken a comprehensive study of the photoluminescence properties of silicon-rich silica and have shown that it is possible to control the peak luminescence wavelength and spectral distribution through careful choice of growth conditions and postprocess annealing. We have identified two mechanisms which are responsible for light emission from this material. These give rise to two luminescence bands: one centered around $2 \mathrm{eV}$, the other between 1.5 and $1.8 \mathrm{eV}$. The annealing behavior of the two bands implies that the higher energy transition is associated with defect centers while the lower energy band is the result of emission from confined excitons within silicon clusters. Fourier transform infrared (FTIR) observation of $\mathrm{Si}-\mathrm{H}$ and $\mathrm{Si}-$ $\mathrm{O}-\mathrm{Si}$ bands yields valuable information on the role of hydrogen desorption and the annealing of oxygen vacancy related defects. We conclude that our results are in broad agreement with those of Shimizu-Iwayama, Komoda, and Fischer et al., and we identify growth conditions which can favor either of the two luminescence mechanisms.

\section{EXPERIMENT}

A number of silicon-rich silica films were deposited on lightly doped $p$-type $\langle 100\rangle$ silicon wafers using PECVD. The sample wafers were first thoroughly cleaned under clean-
TABLE I. Deposition parameters.

\begin{tabular}{cccccc}
\hline \hline & $\begin{array}{c}\mathrm{N}_{2} \mathrm{O}: \mathrm{SiH}_{4} \\
\text { flow rate } \\
(\mathrm{sccm} / 10)\end{array}$ & $\begin{array}{c}\text { rf power } \\
(\mathrm{W})\end{array}$ & $\begin{array}{c}\text { Substrate } \\
\text { temp. } \\
\left({ }^{\circ} \mathrm{C}\right)\end{array}$ & $\begin{array}{c}\text { Chamber } \\
\text { pressure } \\
(\mathrm{mT})\end{array}$ & $\begin{array}{c}\text { Deposition } \\
\text { time } \\
(\mathrm{min})\end{array}$ \\
\hline Sample & $18: 1$ & 10 & 125 & 100 & 60 \\
SS10 & $20: 1$ & 10 & 125 & 100 & 60 \\
SS11 & $22: 1$ & 10 & 125 & 100 & 60 \\
SS12 & $24: 1$ & 10 & 125 & 100 & 60 \\
SS3 & $16: 1$ & 10 & 125 & 100 & 60 \\
SS14 & $14: 1$ & 10 & 125 & 100 & 60 \\
SS24 & $18: 1$ & 10 & 170 & 100 & 120 \\
SS26 & $14: 1$ & 10 & 200 & 100 & 60 \\
SS27 & $18: 1$ & 30 & 200 & 300 & 60 \\
SS28 & $12: 1$ & 10 & 200 & 100 & 60 \\
SS29 & $18: 1$ & 30 & 200 & 100 & 60 \\
\hline \hline
\end{tabular}

room conditions using acetone and isopropyl alcohol followed by sulphuric and nitric acids and finally rinsed with de-ionized water. After placing in the reaction chamber, the wafers were cleaned again with an argon plasma for $5 \mathrm{~min}$. A modified capacitively coupled PlasmaTech PD80 machine was used for the deposition. Precursor gases were a silane/ argon mix [5\% silane $\left(\mathrm{SiH}_{4}\right)$ in argon] and nitrous oxide $\left(\mathrm{N}_{2} \mathrm{O}\right)$ as silicon and oxygen sources, respectively. The nitrous oxide flow rate was fixed at $10 \mathrm{sccm}$ and the silane/ argon mixture flow rate changed from run to run between 240 and $120 \mathrm{sccm}$. A $13.56 \mathrm{MHz}$ rf generator was used to dissociate the precursor gases, the power being varied from run to run between 10 and $30 \mathrm{~W}$. Substrate temperature was set in the range $120-200{ }^{\circ} \mathrm{C}$, and deposition time was either 60 or $120 \mathrm{~min}$. Details of the films deposited are summarized in Table I.

After deposition, each film was divided into a number of pieces which were then annealed under argon for $90 \mathrm{~min}$ at a series of temperatures between 200 and $1000{ }^{\circ} \mathrm{C}$.

Photoluminescence spectra were measured for each film using a Coherent argon-ion laser operating at 351, 457, or $476 \mathrm{~nm}$ as the excitation source. For measurement of roomtemperature spectra, samples were in air, while for measurement of spectra at low temperatures samples were placed in a Janis Supertran continuous-flow liquid nitrogen cryostat. Temperature control of $0.1 \mathrm{~K}$ between 70 and $300 \mathrm{~K}$ was achieved using a LakeShore 321 temperature controller and heater. Spectra were dispersed using a Bentham M300 monochromator and detection was via a Bentham 231 side window photomultiplier tube, a current preamplifier, and lock-in amplifier. Both the detection electronics and monochromator were computer controlled; care was taken to ensure that the spectra were as accurate and repeatable as possible. A diffraction grating was used as a premonochromator to filter out plasma lines from the laser and a Schott glass filter (OG495) placed immediately following the collection optics served to prevent the scattered laser line entering. A standard Bentham IL1 stabilized tungsten bulb white-light source was used to calibrate the entire optical system of collection lenses, monochromator, and photomultiplier tube. It was found that over the range studied the system response was very sensitive to the nonlinearity of response of both the photomultiplier tube and the monochromator grating. We 
TABLE II. Film composition and thickness measured by SIMS.

\begin{tabular}{lcccccc}
\hline \hline Sample & $\begin{array}{c}\mathrm{Si}(\mathrm{O}) \\
(\text { at. \%) }\end{array}$ & $\begin{array}{c}\mathrm{Si}(\mathrm{e}) \\
(\text { at. \%) }\end{array}$ & $\begin{array}{c}\mathrm{O} \\
(\text { at. \%) }\end{array}$ & $\begin{array}{c}\mathrm{N} \\
\text { (at. \%) }\end{array}$ & $\begin{array}{c}\mathrm{C} \\
(\text { at. \%) }\end{array}$ & $\begin{array}{c}\text { Thickness } \\
(\mu \mathrm{m})\end{array}$ \\
\hline SS24 & 28.8 & 8.5 & 56.6 & 4.6 & 1.6 & 3.1 \\
SS26 & 33.3 & 0.2 & 54.6 & 6.3 & 5.6 & 0.25 \\
SS27 & 30.9 & 0.8 & 62.8 & 4.8 & 0.8 & 1.0 \\
SS28 & 27.0 & 8.4 & 55.1 & 6.0 & 3.5 & 0.38 \\
SS29 & 22.4 & 19.3 & 49.6 & 6.9 & 1.9 & 0.27 \\
\hline \hline
\end{tabular}

cannot stress too strongly how important it is to carry out a thorough study of the system response and to correct spectra accordingly. All too often, spectra are presented in the literature which are not corrected for system response. In the case of spectra as broad as those reported here, it is almost impossible to draw any conclusions about underlying mechanisms on the basis of uncorrected data, as peak positions, linewidths, and peak shapes can change enormously when the system response has been removed. Particular attention must be paid to the long wavelength region of the spectrum, as the photomultiplier tube response is highly nonlinear below $1.7 \mathrm{eV}$. In this case, care was also taken to eliminate any contribution to the spectra from photoluminescence induced in the Schott glass filter by the scattered laser light. Although weak, photoluminescence from the filter could be a problem when studying weak photoluminescence spectra. The filter was placed immediately adjacent to the last lens of the collection optics, at which point the scattered laser light was diffuse and unfocused.

In order to study defects within the films, a number of samples, both as-deposited and postannealing, were studied using ESR. Scans were centered around $g=2$.

In order to monitor the chemical changes within the films brought about by annealing, selected films were studied using FTIR. This allowed the determination of such changes as loss of hydrogen (i.e., reduction in $\mathrm{Si}-\mathrm{H}_{x}$ signal) and increase in oxidation state of silicon (i.e., increase in $\mathrm{Si}-\mathrm{O}-\mathrm{Si}$ signal). The instrument used was an FTIR microscope with a resolution of approximately $80 \mu \mathrm{m}$.

Samples of as-grown films were analyzed for chemical composition using both secondary ion mass spectroscopy (SIMS) and electron-probe microanalysis. In particular, we were interested in the ratio between elemental and oxidized silicon. This was readily obtained from SIMS data. Film thicknesses for selected samples were measured using Talystep.

\section{RESULTS}

\section{A. Thickness and composition}

Table I shows the deposition parameters for the samples studied. In those cases in which the composition was measured by SIMS, the results are tabulated in Table II along with thicknesses. It is interesting to note the large variations in film thickness, indicating very different growth rates. In particular, although the deposition time for SS24 is twice that of the other films, its thickness is three times that of SS27 and ten times that of the remaining three. It should be noted that film SS27 was in fact very inhomogeneous and appeared to include large $(\sim 250 \mu \mathrm{m})$ particulates, presumably amorphous silicon. This may be a consequence of the high pressure in the deposition chamber during film growth.

\section{B. Photoluminescence}

Figure 1 shows photoluminescence spectra for sample SS24 both as-grown and following annealing at $T_{a}=400{ }^{\circ} \mathrm{C}$. The presence of strong thin-film interference fringes in the spectra serves to confuse the interpretation, so subsequent figures have had fringes removed using a smoothing algorithm. Figure 2 shows spectra for sample SS24 following anneals at $300,400,500$, and $600{ }^{\circ} \mathrm{C}$. Figure 3 shows spectra from the same sample for $T_{a}=600,800$, and $1000^{\circ} \mathrm{C}$. All intensities are normalized with respect to the $400{ }^{\circ} \mathrm{C}$ annealed sample and the data are shown on two separate graphs for clarity. Figures 4 and 5 show similar spectra for samples SS26 and SS28. Again, all intensities are normalized to the $400{ }^{\circ} \mathrm{C}$ annealed sample of SS24 and are therefore directly comparable. In a number of cases there appear to be two distinct bands in the photoluminescence spectra which are separated by up to $0.5 \mathrm{eV}$.

\section{Fourier transform infrared}

Figure 6 shows FTIR data for sample SS26. Spectra are presented for unannealed, $300{ }^{\circ} \mathrm{C}$ annealed, and $400{ }^{\circ} \mathrm{C}$ annealed samples. For sample $\mathrm{SS} 24$, the $\mathrm{Si}-\mathrm{H}_{x}$ band around $2200 \mathrm{~cm}^{-1}$ is magnified in the inset. Table III gives the as-

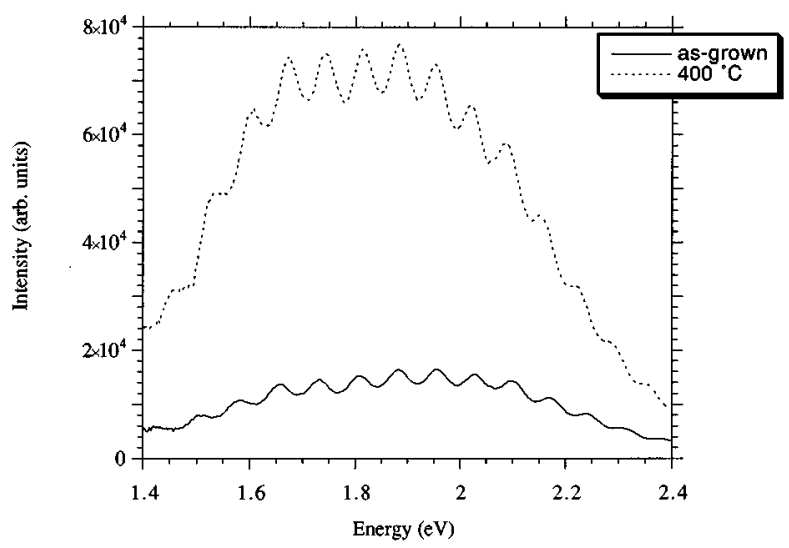

FIG. 1. Room-temperature photoluminescence spectra of portions of sample SS24 as-grown and following a $400{ }^{\circ} \mathrm{C}$ anneal. 


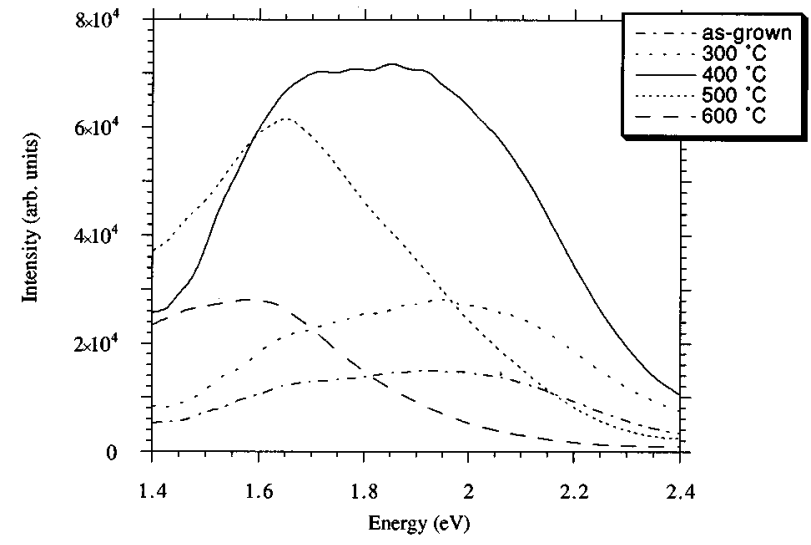

FIG. 2. Smoothed room-temperature photoluminescence spectra of sample $\mathrm{SS} 24$ as a function of annealing temperature.

signments of FTIR bands observed in $\mathrm{SiO}_{x}$ samples. The bands around $2200 \mathrm{~cm}^{-1}$ associated with $\mathrm{Si}-\mathrm{H}_{x}$ stretches are shifted up in energy by approximately $100 \mathrm{~cm}^{-1}$ with respect to the corresponding bands in hydrogenated amorphous silicon. This can be ascribed to the effect of the $\mathrm{SiO}_{2}$ matrix around the $\mathrm{Si}-\mathrm{H}$ sites. In particular, it is worth noting that for SS24 the $2200 \mathrm{~cm}^{-1}$ band shows some evidence of three separate bands at 2150, 2200, and $2250 \mathrm{~cm}^{-1}$. From comparison with Petrova-Koch et al.'s work on FTIR of porous silicon, ${ }^{29}$ these can be assigned as $\mathrm{Si}-\mathrm{H}, \mathrm{Si}-\mathrm{H}_{2}$, and $\mathrm{Si}-\mathrm{H}_{3}$ stretches, respectively. Furthermore, from the same article, it can be inferred that the separation of the $\mathrm{Si}-\mathrm{H}_{x}$ band into its three components implies "large", clusters $(>20 \AA$ diam) of silicon with hydrogen terminations. Conversely, the broadening of the bands by the local electric field around "small" clusters $(<20 \AA$ diameter $)$ tends to lead to spectra with a broad $\mathrm{Si}-\mathrm{H}_{x}$ band. We may expect further inhomogeneous broadening of the FTIR bands by the presence of the extended $\mathrm{SiO}_{2}$ matrix, so this estimate of the transition between small and large clusters is at best a rough estimate. However, it is reasonable to conclude that if the $\mathrm{Si}-\mathrm{H}_{x}$ band is not resolvable into its three components, then our samples con-

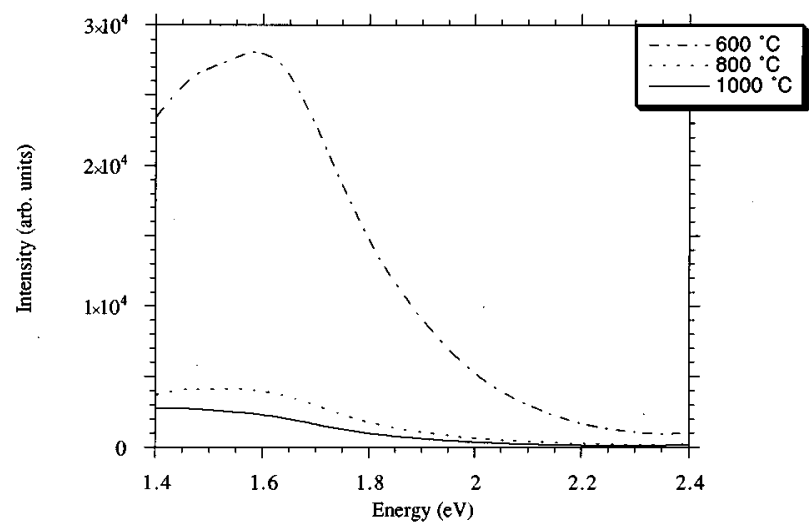

FIG. 3. Smoothed room-temperature photoluminescence spectra of sample SS24 as a function of annealing temperature.

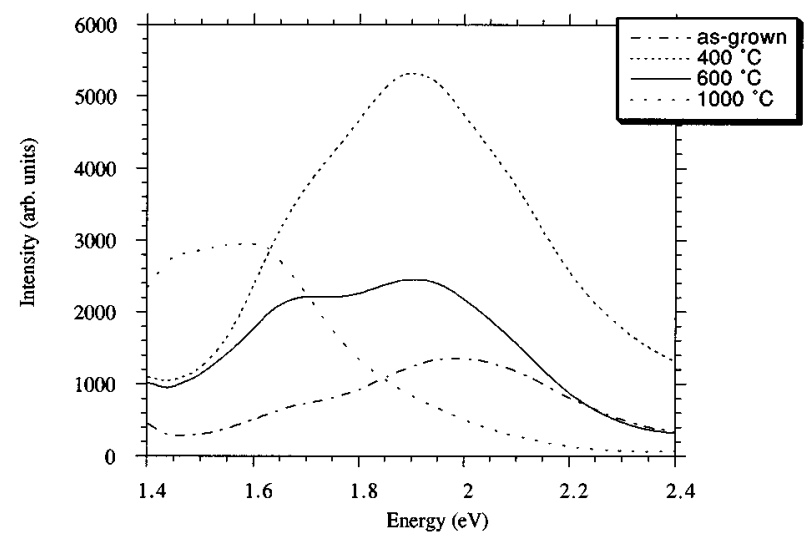

FIG. 4. Smoothed room-temperature photoluminescence spectra of sample SS26 as a function of annealing temperature.

tain silicon clusters which are of the same order of magnitude as Petrova-Koch's estimate, i.e., less than $100 \AA$ in diameter.

Figure 7 shows the dependence of the intensity of the FTIR bands at $2200 \mathrm{~cm}^{-1}\left(\mathrm{Si}-\mathrm{H}_{x}\right), 1100 \mathrm{~cm}^{-1}(\mathrm{Si}-\mathrm{O}-\mathrm{Si})$, and $1025 \mathrm{~cm}^{-1}(\mathrm{Si}-\mathrm{O}-\mathrm{Si})$ on annealing temperature for sample SS26. There is a clear reduction in the hydrogen content of the film as $T_{a}$ increases. The $\mathrm{Si}-\mathrm{H}_{x}$ band has completely disappeared following the $1000{ }^{\circ} \mathrm{C}$ anneal. Accompanying this is an increase in both of the $\mathrm{Si}-\mathrm{O}-\mathrm{Si}$ bands up to $T_{a}=600{ }^{\circ} \mathrm{C}$, implying an annealing out of oxygen vacancies. It is not clear what the cause of the subsequent decease in these bands at $T_{a}=1000{ }^{\circ} \mathrm{C}$ is, but it could possibly be due to growth of silicon clusters.

\section{Electron spin resonance}

Figure 8 shows ESR spectra for a control sample of cut silicon and similar sized pieces of sample SS26 annealed over a range of temperatures. The presence of a strong signal around $g=2.005$ in the latter and its absence in the former suggests that this signal is not due to dangling bonds formed by cleaving the silicon substrate, but is from paramagnetic defects associated with oxygen vacancies in the film. A $g$ value of 2.0055 is generally taken as a diagnostic of the presence of $P_{b}$ centers in silica, or their analogue in amorphous silicon, the $D$ center. Both of these defects consist of an unpaired spin located on a silicon atom bonded to three further silicon atoms $\left(\mathrm{Si}_{3} \equiv \mathrm{Si} \uparrow\right)$. In the case of amorphous silicon, such defects are characterized as dangling bonds at the surface, ${ }^{30}$ while in the case of silica films on silicon, $P_{b}$ centers can often be associated with oxygen vacancies at the

TABLE III. FTIR bands and assignments.

\begin{tabular}{cc}
\hline \hline $\begin{array}{c}\text { Frequency } \\
\left(\mathrm{cm}^{-1}\right)\end{array}$ & Assignment \\
\hline 1050 & $\mathrm{Si}-\mathrm{O}-\mathrm{Si}$ \\
1100 & $\mathrm{Si}-\mathrm{O}-\mathrm{Si}$ \\
2150 & $\mathrm{Si}-\mathrm{H}$ \\
2200 & $\mathrm{Si}-\mathrm{H}_{2}$ \\
2250 & $\mathrm{Si}-\mathrm{H}_{3}$ \\
\hline
\end{tabular}




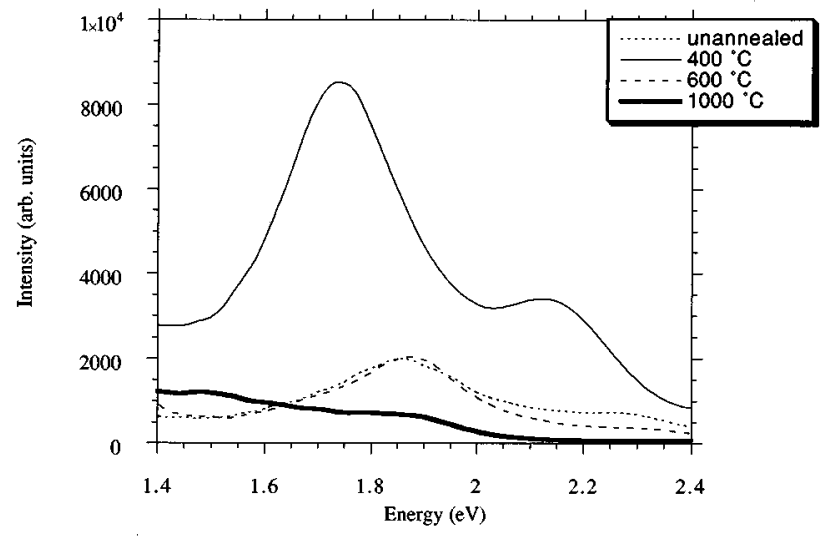

FIG. 5. Smoothed room-temperature photoluminescence spectra of sample SS28 as a function of annealing temperature.

$\mathrm{Si} / \mathrm{SiO}_{2}$ interface. ${ }^{30,31}$ It may therefore be the case that the $P_{b}$ centers in our samples are located at the cluster/silica interface. Studies of the passivation and depassivation of $P_{b}$ centers in thin film $\mathrm{SiO}_{2}$ have demonstrated an increase in $P_{b}$ content on annealing at $T>500{ }^{\circ} \mathrm{C}$ in vacuum, argon, nitrogen, and air. ${ }^{30}$ Further annealing at higher temperatures can reduce defect concentration by compensating dangling bonds with interstitial oxygen and water. The rapid increase in ESR signal above $T=500{ }^{\circ} \mathrm{C}$ agrees well with other observations of the annealing behavior of the $P_{b}$ center. The decrease between $T=200$ and $500{ }^{\circ} \mathrm{C}$ may be due to an initial uptake of interstitial hydrogen.

\section{E. Low-temperature photoluminescence}

Figure 9 presents photoluminescence spectra obtained at 70 and $300 \mathrm{~K}$ for sample SS24 following a $400{ }^{\circ} \mathrm{C}$ anneal. On cooling to cryogenic temperatures an increase in the peak intensity of luminescence by a factor of around 8.5 can clearly be seen. It is the case for all films studied that there was some increase in photoluminescence intensity on cooling, but the magnitude of this varied considerably from approximately 3 (SS27) to around 8.5 (SS24). Figure 10 shows photoluminescence spectra from sample SS24 obtained at 70, 200, 250, and $300 \mathrm{~K}$. All spectra are normalized to give peak intensities of 1 . A blueshift in the higher energy peak with increasing temperature is evident, although it is not clear how the lower energy transition is affected. To investigate this further, spectra of SS24 taken over the range 70$300 \mathrm{~K}$ were fitted using two Gaussian components. Figure 11 illustrates the shifts in peak energy of the two components with temperature; Fig. 12 shows the temperature dependence of the photoluminescence intensity. Over the range of temperatures studied, the temperature quenching of the luminescence appears more significant for the higher energy band. In particular, the results for the higher energy transition shown in Fig. 11 are strikingly similar to those presented by Fischer et al. ${ }^{17}$

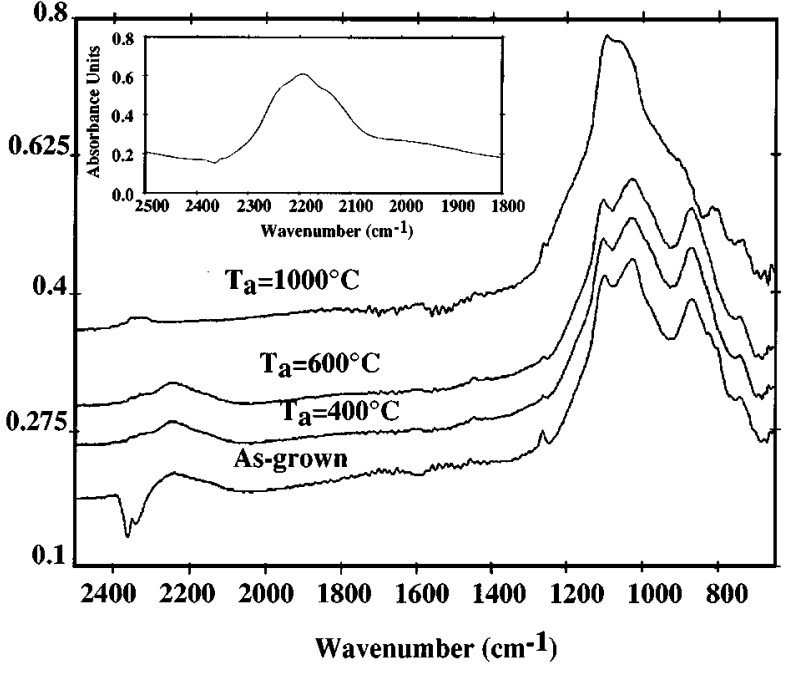

FIG. 6. FTIR absorption spectra for sample SS26 as-grown and following 400,600 , and $1000{ }^{\circ} \mathrm{C}$ anneals. Inset shows the region around $2200 \mathrm{~cm}^{-1}$ magnified for sample SS24.

\section{DISCUSSION}

Looking first at the photoluminescence results, the situation appears to be complex. Although all the films studied show broadband emission, not all show bright luminescence as-grown (SS27, for example). Some appear to exhibit a pronounced redshift of the luminescence peak on annealing (SS24,SS26) while others show this only weakly (SS29). Some films show strong quenching of luminescence when annealed at temperatures above $600{ }^{\circ} \mathrm{C}$ (SS24,SS26) while others only begin to exhibit luminescence when annealed at temperatures in excess of this (SS27). This diversity of results strongly suggests the presence of more than one luminescence mechanism. From inspection of the smoothed data, it is clear in most cases that the spectra observed exhibit contributions from two luminescence bands. This is most pronounced for sample SS28 but does seem to be the case for the remaining samples as well. Figure 13 shows roomtemperature photoluminescence data for sample SS24C (annealed at $400{ }^{\circ} \mathrm{C}$ ) resolved into two Gaussian components. This shows bands centered around 1.95 and $1.7 \mathrm{eV}$.

Looking in more detail at Fig. 13 and bearing in mind the separation of the spectra into two bands, it becomes possible to make an analysis of the effects of annealing. The as-grown film shows predominantly $1.95 \mathrm{eV}$ emission with a shoulder at $1.75 \mathrm{eV}$. On annealing to $400{ }^{\circ} \mathrm{C}$, both bands increase in intensity by a factor of around 7 and the shape of the spectrum remains largely unchanged. However, after annealing at $600{ }^{\circ} \mathrm{C}$, the $1.95 \mathrm{eV}$ band falls rapidly while the $1.75 \mathrm{eV}$ band exhibits a redshift of around $0.05 \mathrm{eV}$. Further annealing of the sample to $1000^{\circ} \mathrm{C}$ results in complete quenching of the $1.95 \mathrm{eV}$ band, attenuation of the lowenergy band but, more significantly, a further redshift of 0.3 $\mathrm{eV}$.

As a general trend, this applies to all the samples studied: the higher energy band, if present, remains at a fixed energy around $2 \mathrm{eV}$ regardless of annealing temperature and becomes completely quenched at temperatures above 600- 


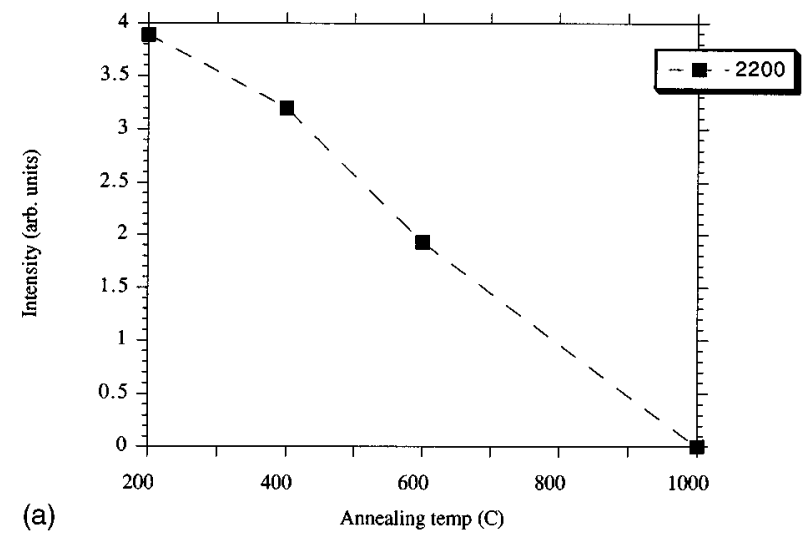

(a)

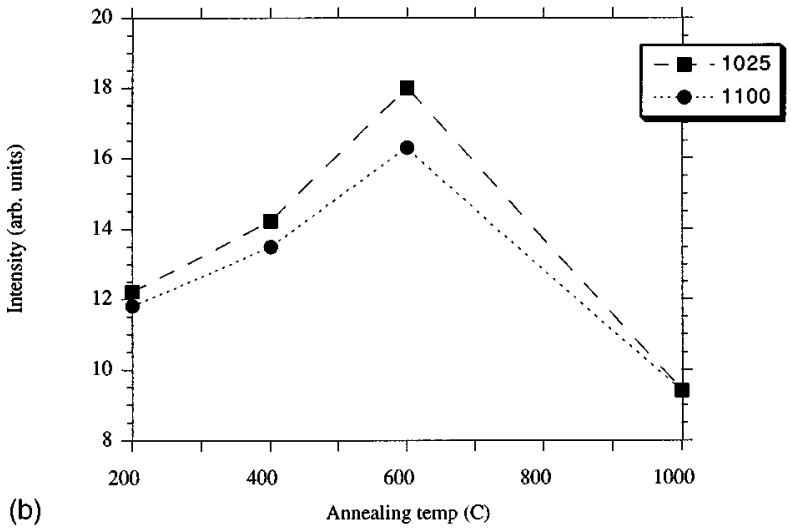

FIG. 7. Dependence of intensities of 2200, 1100, and $1025 \mathrm{~cm}^{-1}$ FTIR lines on annealing temperature for sample SS26.

$800{ }^{\circ} \mathrm{C}$. The lower energy band is present even in samples annealed at temperatures up to $1000{ }^{\circ} \mathrm{C}$ and shows a redshift with annealing, the magnitude of which varies from sample to sample. Sample SS29 shows a variation of this pattern: the $2 \mathrm{eV}$ band is rather weak even at $T_{a}=400{ }^{\circ} \mathrm{C}$. The $1.7 \mathrm{eV}$ band grows rapidly as $T_{a}$ approaches $400{ }^{\circ} \mathrm{C}$, after which it quickly becomes quenched and shows little evidence of an annealing-related redshift. However, the two transitions are clearly present.

Turning to the question of determining the mechanisms responsible for the two photoluminescence bands, perhaps the first point to address is the redshift of the low-energy band. It has been well established by a number of groups that annealing silicon-rich silica films at temperatures in excess of $600{ }^{\circ} \mathrm{C}$ results in accretion of excess silicon atoms and aggregates to form progressively larger clusters. ${ }^{12}$ If annealed at a high enough temperature for a sufficient time, the larger silicon clusters will tend to "swallow up" the smaller ones, resulting ultimately in macroscopic inclusions of "bulk" silicon within the $\mathrm{SiO}_{2}$ matrix. This is referred to as "Ostwald ripening.", 17 An increase in cluster size produces a reduction in confinement energy and therefore a narrowing of the optical band gap. Observation of annealing-related redshifts in luminescence spectra is therefore generally taken to be good evidence in support of the quantum confinement hypothesis. The dependence of the photoluminescence energy on cluster size is well established and formulae have been published which quantify the relationship between cluster size and band-gap energy. ${ }^{3,32-34}$ From these, it is sug-

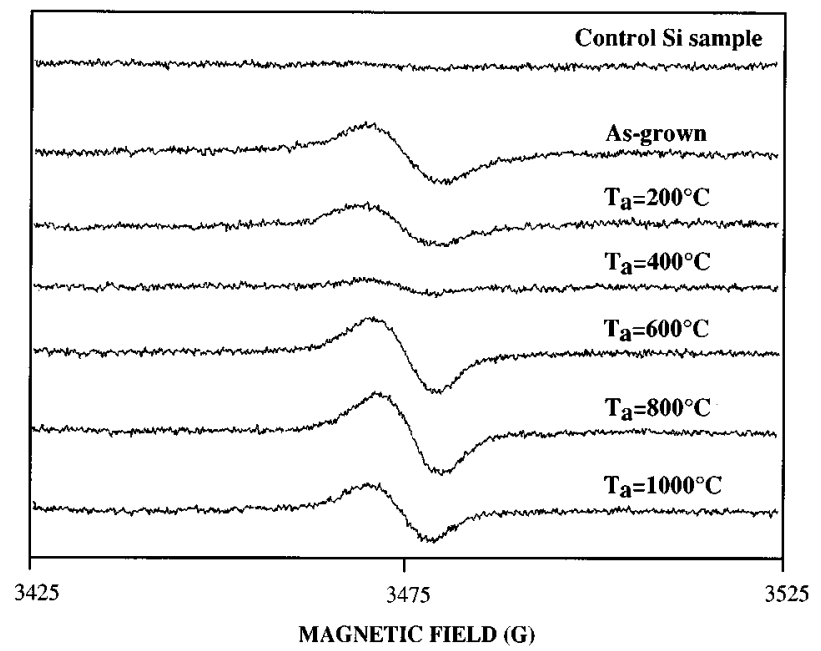

FIG. 8. ESR spectra for segments of sample SS26 as-grown and following anneals at a number of temperatures in the range $200-1000{ }^{\circ} \mathrm{C}$. The top trace is for a control sample of silicon cut to the same size.

gested that a peak luminescence emission around $1.7 \mathrm{eV}$ corresponds to cluster dimensions of approximately $4 \mathrm{~nm}$. The quantum confinement model also predicts a strong increase in luminescence intensity with decreasing cluster size. This may help to explain the reduction in photoluminescence intensity accompanying the redshift of the low-energy band on annealing at $T_{a}>600{ }^{\circ} \mathrm{C}$.

For the case of the high-energy band, the absence of an annealing-related redshift makes it unlikely that this band is the result of recombination of confined excitons. However, luminescence around 1.9 and $2 \mathrm{eV}$ has been noted before in both amorphous silica and quartz. This is generally associated with defect states produced by implantation or irradiation. Shimizu-Iwayama's work suggested the presence of defects in implanted samples and assigned an emission band around $2.2 \mathrm{eV}$ to defect luminescence from states at the silicon cluster $\mathrm{SiO}_{2}$ interface.

Our ESR data do indicate the presence of a significant number of paramagnetic centers in all the films studied. The signal at $g=2.005$ is associated with the $P_{b}$ oxygen vacancy

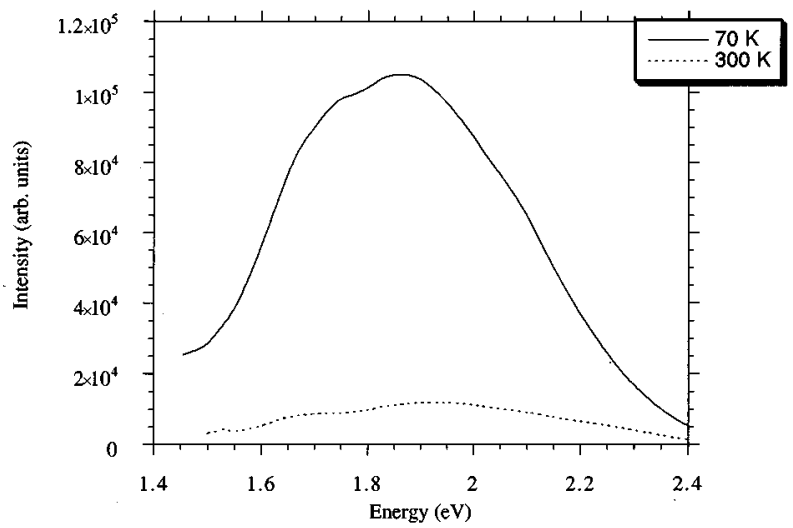

FIG. 9. Smoothed photoluminescence spectra of sample SS24 obtained at 70 and $300 \mathrm{~K}$. 


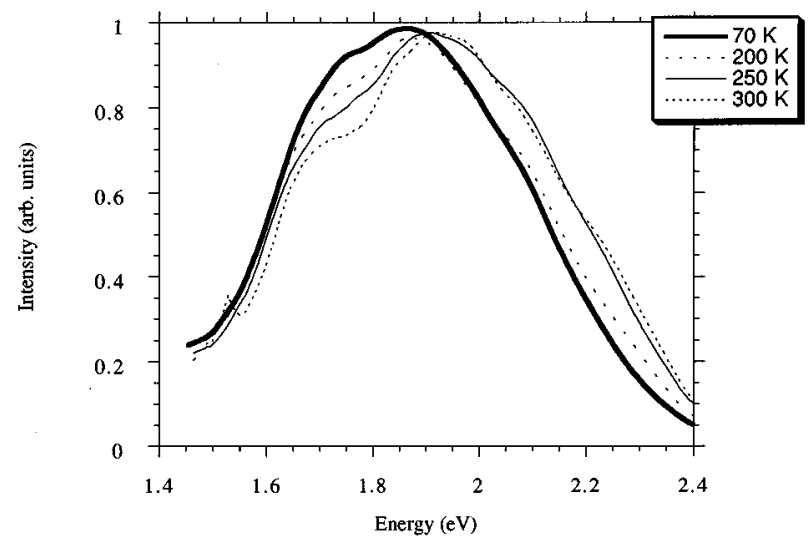

FIG. 10. Smoothed normalized photoluminescence spectra of sample SS24 as a function of temperature.

defect and is present in all the as-grown and annealed samples. Looking at sample SS24, we observe a concentration of $5 \times 10^{16}$ spins per gram in the as-grown film. However, comparison of the annealing behavior of the $2 \mathrm{eV} \mathrm{lu}-$ minescence band with that of the $P_{b}$ centers shows that it is unlikely that these defects are responsible for the emission. For sample SS26, between 200 and $600{ }^{\circ} \mathrm{C}$, the photoluminescence signal increases by a factor of 2 while the ESR signal at $g=2.0055$ falls sharply. Annealing at temperatures in excess of $600{ }^{\circ} \mathrm{C}$ leads to complete quenching of the $2 \mathrm{eV}$ band but this is the range over which the ESR signal is strongest.

There has been considerable debate in the literature over the origin of the 1.9 and $2 \mathrm{eV}$ luminescence bands in silica, ${ }^{34,35}$ but the current model suggests that they are due to emission from nonbridging oxygen hole centers $(\mathrm{NBOHCs})^{34}$ Such defects are usually associated with the radiolysis of hydroxyl groups or the cleavage of strained silicon-oxygen bonds by irradiation:

$$
\begin{aligned}
& \equiv \mathrm{Si}-\mathrm{O}-\mathrm{H} \rightarrow \equiv \mathrm{Si}-\mathrm{O} \uparrow+\mathrm{H} \cdot \\
& \equiv \mathrm{Si}-\mathrm{O}-\mathrm{Si} \equiv \longrightarrow \equiv \mathrm{Si}-\mathrm{O} \uparrow+\mathrm{Si} \equiv
\end{aligned}
$$

However, there is evidence of the presence of NBOHCs in as-grown CVD oxide films. ${ }^{36}$ It is puzzling that we do not

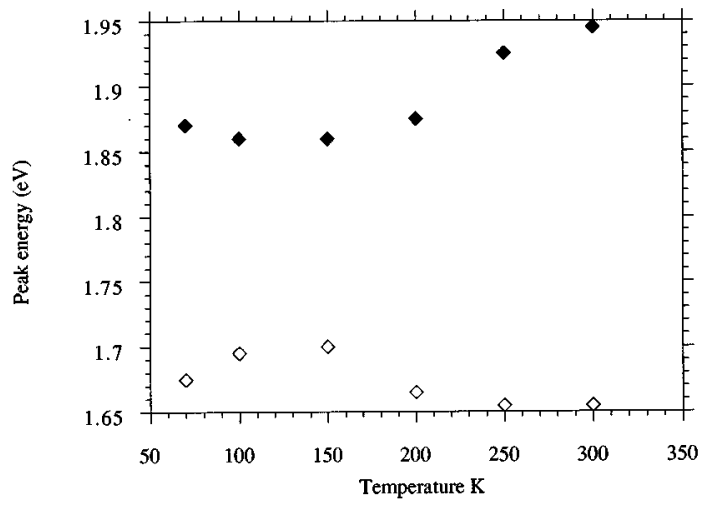

FIG. 11. Peak energy as a function of temperature for sample SS24. Upper points: data for higher energy band. Lower points: data for low-energy band.

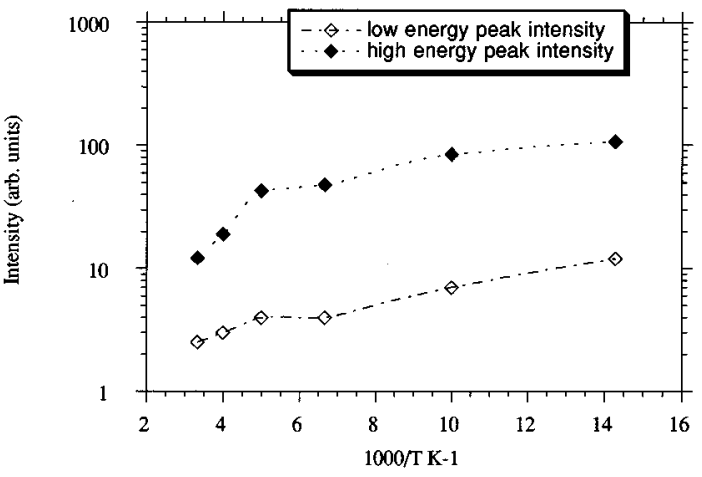

FIG. 12. Photoluminescence intensity as a function of temperature for sample SS24. Intensities for the two bands obtained by fitting data with two Gaussian transitions.

see the characteristic ESR signal due to the NBOHC at $g=2.001$. It may thus be that some other, nonparamagnetic defect is responsible for the $2 \mathrm{eV}$ band. Certainly, on the basis of our data, we cannot conclusively identify it. There is some evidence that the electron trap $\mathrm{Si}_{3}$ defect luminesces at these energies, ${ }^{34}$ but the presence of this defect in our samples remains speculation at present. However, the annealing behavior and lack of redshift in the case of the $2 \mathrm{eV}$ band suggests a defect origin.

The observation that in all cases the $2 \mathrm{eV}$ photoluminescence band is completely quenched following anneals in excess of $600{ }^{\circ} \mathrm{C}$ indicates that the quenching mechanism is tied in with the filling of oxygen vacancies in the silica matrix by diffusion of interstitial oxygen and water. However, the low-temperature anneal behavior of the $2 \mathrm{eV}$ band requires further investigation.

Looking at the FTIR data, two processes become apparent. First, all the samples studied are initially rich in hydrogen to varying degrees, as illustrated by the presence of the $\mathrm{Si}-\mathrm{H}_{x}$ stretch band at $2200 \mathrm{~cm}^{-1}$. Annealing the films at progressively higher temperatures causes a reduction in the intensity of this band, as is consistent with the evolution of hydrogen until, following a $600{ }^{\circ} \mathrm{C}$ anneal, the band is in all cases below our detection limit. At the same time, there is a gradual increase in the $\mathrm{Si}-\mathrm{O}-\mathrm{Si}$ bands at 1100 and 1025

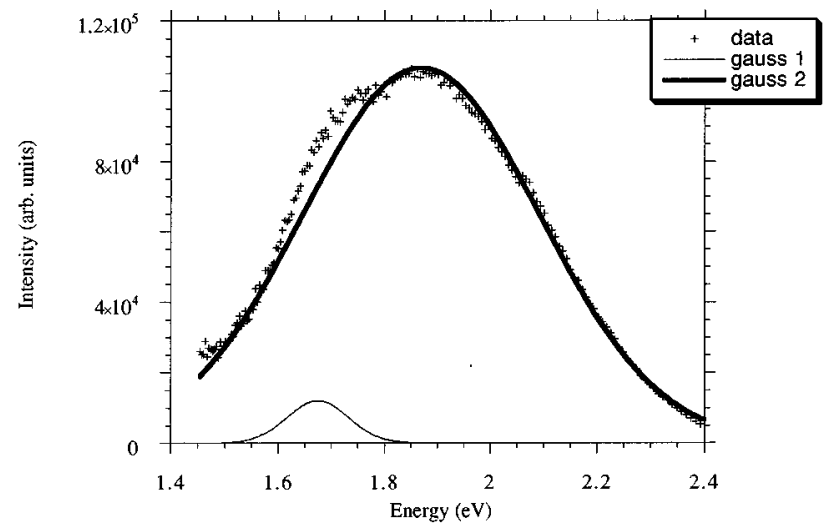

FIG. 13. Double Gaussian fit to photoluminescence data from sample SS24. 
$\mathrm{cm}^{-1}$, indicating an annealing out of oxygen vacancies as sufficient thermal energy is delivered to interstitial oxygen atoms for them to diffuse to fill vacancies. The subsequent decrease in these bands at $T_{a}>1000{ }^{\circ} \mathrm{C}$ may be due to the growth of silicon clusters and hence the formation of $\mathrm{Si}-\mathrm{Si}$ bonds in preference to $\mathrm{Si}-\mathrm{O}$ bonds.

It is worth noting that in almost all cases studied, the $\mathrm{Si}-\mathrm{H}_{x}$ band around $2200 \mathrm{~cm}^{-1}$ was not resolvable into the three components. This implies that the silicon clusters being studied are less than $100 \AA$ in diameter.

It would initially be expected that removal of hydrogen from hydrogen-rich $\mathrm{SiO}_{x}$ films would produce a large number of dangling bonds which would in turn act as nonradiative recombination centers and quench luminescence. ${ }^{37}$ However, there is a clear increase in the photoluminescence intensity from the $2 \mathrm{eV}$ band following annealing at $T_{a}<600{ }^{\circ} \mathrm{C}$. FTIR data certainly indicate almost complete removal of hydrogen by this point. This suggests that the evolution of hydrogen from the films at $T_{a}<600{ }^{\circ} \mathrm{C}$ serves to increase the population of optically active defects at a rate which is greater than that of their annihilation by thermally excited interstitial oxygen atoms. This is perhaps unsurprising when the differences in mobility between oxygen and hydrogen are considered. However, the nature of the defect responsible for the $2 \mathrm{eV}$ band is not clear. We assume that it is produced by the cleavage of $\mathrm{Si}-\mathrm{H}$ or $\mathrm{O}-\mathrm{H}$ bonds and may be generically similar to the NBOHC, although it does not appear to be ESR active.

The dangling bonds left by hydrogen evolution exist in a very different environment from that seen by similar dangling bonds in either porous or amorphous silicon. In the case of $\mathrm{SiO}_{x}$, the presence of the extended $\mathrm{SiO}_{2}$ network around the dangling bond sites means that they cannot be considered in isolation as nonradiative recombination centers. The conventional picture is that an increase in dangling bonds leads to a sharp decrease in photoluminescence; ${ }^{37}$ this is known to be very much the case in both amorphous and porous silicon. ${ }^{38,39}$ In both materials the presence of even a small number of dangling bonds serves to completely quench photoluminescence. However, it appears that for the particular case of silicon-rich silica, the defect centers produced by annealing and associated hydrogen evolution act as radiative recombination sites.

Figure 14 illustrates a suggested scheme for the formation of defect centers in $\mathrm{SiO}_{x}$ films by hydrogen evolution. Figure 14(a) shows the initial hydrogenated silicon-rich silica matrix. Heating this at $T_{a}<500{ }^{\circ} \mathrm{C}$ rapidly drives off hydrogen, leaving silicon dangling bonds [Fig. 14(b)]. Further heating at $T_{a}>500{ }^{\circ} \mathrm{C}$ allows interstitial and nonbonded oxygen atoms and water molecules to diffuse to the oxygen vacancy sites [Fig. 14(c)], leading eventually to full compensation of the dangling bonds [Fig. 14(d)]. At this point, the existing silicon clusters may begin to grow as interstitial silicon atoms diffuse.

Although all films studied exhibit broadly similar photoluminescence spectra, i.e., bands at $2 \mathrm{eV}$ and $1.5-1.75 \mathrm{eV}$, the relative magnitudes and absolute positions of the two bands vary considerably. Some samples show only a weak contribution from the $2 \mathrm{eV}$ band while others exhibit pre-

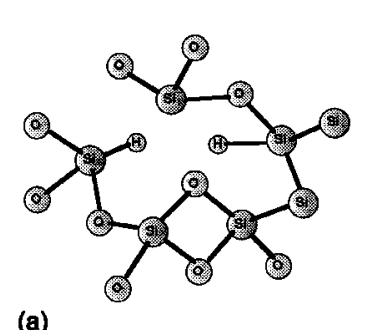

(a)

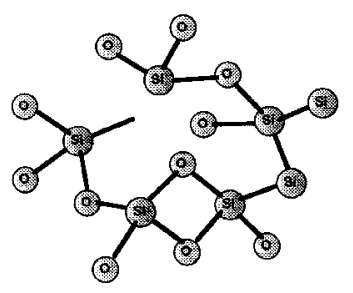

(c)

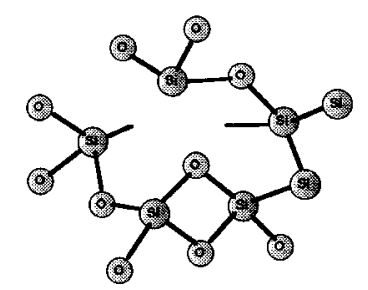

(b)

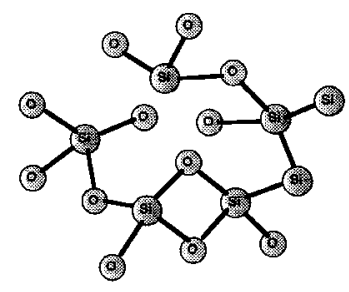

(d)
FIG. 14. Scheme for creation of dangling bonds by thermal evolution of hydrogen followed by compensation by diffusion of interstitial oxygen. The sequence runs from low to high annealing temperature showing: (a)-(b): rapid removal of hydrogen and (c)-(d): slow diffusion of oxygen to resultant dangling bond centers.

dominantly defect luminescence with little signal from silicon clusters. The FTIR results indicated that those films which were initially very hydrogen rich were those which showed the greatest contribution from defect luminescence and the greatest increase in the $2 \mathrm{eV}$ luminescence band for $T_{a}<500{ }^{\circ} \mathrm{C}$. These tended to be those films which were grown at low rf power and high chamber pressure. Under these conditions more hydrogen is likely to be incorporated into the growing film as a result of less complete dissociation of silane. Conversely, those grown at higher powers and lower pressures showed a stronger contribution from the 2 $\mathrm{eV}$ band. This suggests the possibility of controlling the photoluminescence properties of $\mathrm{SiO}_{x}$ through careful choice of growth parameters. "True" quantum confinement effects only appear to be dominant for films which contain few intrinsic defects, i.e., those which are initially low in hydrogen or those which have been annealed at $T_{a}>500{ }^{\circ} \mathrm{C}$. Conversely, the brightest visible luminescence is obtained from defect-rich films. This may help to clarify some of the confusion surrounding the contradictory reports of some $\mathrm{SiO}_{x}$ films exhibiting photoluminescence as-grown, and others only showing this following relatively high temperature anneals. It may be the case that films grown by those groups who report no luminescence as-grown contain very little hydrogen, and consequently exhibit no defect photoluminescence. Assuming the excess silicon to be evenly distributed in these samples, luminescence from quantum confinement of excitons will only be seen when the samples are heated to temperatures sufficient for silicon clusters to aggregate.

It should be borne in mind that the peak energy and intensity of the low-energy luminescence band are not necessarily determined by the amount of excess silicon present in a film. What is more important is the size distribution of silicon aggregates. The excess silicon may be present as very 
widely distributed small inclusions or as a smaller number of large clusters. The two cases will lead to very different photoluminescence spectra and annealing behavior. The luminescence properties of films are therefore not necessarily determined by overall film stoichiometry: differences in growth conditions (i.e., rf power, chamber pressure, substrate temperature, etc.) can produce very different films with the same elemental composition.

The photoluminescence spectra of all samples show a temperature quenching effect, albeit of a varying degree. Sample SS24 showed the most significant change, with a reduction in integrated luminescence intensity of around 8.5 on warming from 70 to $300 \mathrm{~K}$; that seen in other samples was sometimes significantly smaller than this. The shift in luminescence peak energies with temperature is significant: the defect-associated band exhibits behavior very similar to that reported by Fischer et al. which has been associated with highly localized radiative recombination centers in the bulk matrix. ${ }^{17} \mathrm{~A}$ blueshift of this type is normally associated with the presence of a strong nonradiative recombination process in the bulk material. The absence of such a blueshift in the case of the lower energy band implies a much weaker nonradiative path.

\section{CONCLUSIONS}

We have shown that the photoluminescence from silicon-rich silica films is the result of two separate luminescence mechanisms: defect luminescence, possibly from nonbridging oxygen centers or related oxygen vacancies, and radiative recombination of confined excitons. Our results are in broad agreement with those of Shimizu-Iwayama, Komoda, and Fischer et al. We have proposed a mechanism for defect production within hydrogen-rich $\mathrm{SiO}_{x}$ films which involves evolution of hydrogen at a faster rate than the diffusion of interstitial oxygen to compensate the resultant dangling bonds. Evidence for this mechanism comes from photoluminescence and FTIR data which indicate a decrease in hydrogen content accompanied by an increase in photoluminescence intensity for $T_{a}<600{ }^{\circ} \mathrm{C}$. Above this temperature, annealing out of the oxygen vacancies leads to a reduction in defect luminescence and a growth of silicon clusters as reflected in a redshift of the low-energy photoluminescence peak. However, we are unable at this stage to conclusively identify the luminescent defects as NBOHCs. This scheme may help to explain some of the apparently contradictory photoluminescence results published elsewhere. Depending on the film growth technique, it is possible to produce $\mathrm{SiO}_{x}$ films which exhibit primarily either defect luminescence or that associated with exciton confinement in silicon clusters. In the latter case, a redshift of the photoluminescence peak with annealing may be readily observed, dependent on growth conditions, while in the former, annealing above $T_{a}=500{ }^{\circ} \mathrm{C}$ will simply serve to quench the signal. Also, due to the very broad nature of both bands and the presence of thin-film fringes in many spectra, in some cases it requires care to separate the contributions of the two mechanisms to photoluminescence data.

If silicon-rich silica is to be a viable optoelectronic material for use in such applications as displays, optical ampli- fiers, and filters etc., then a solid understanding of $\mathrm{SiO}_{x}$ luminescence mechanisms is a prerequisite. The aim of this study was to attempt to reduce some of the confusion surrounding the optical properties of this material, although there are still a number of questions to be addressed. In particular, the precise nature of the defect responsible for the 2 $\mathrm{eV}$ emission remains to be identified.

\section{ACKNOWLEDGMENTS}

The authors thank the Optoelectronics Interdisciplinary Research Center (Southampton) and the Engineering and Physical Sciences Research Council for funding. They are grateful to ISST (Loughborough) for the Auger analysis, Dr. H. J. Milledge of the UCL Department of Geological Sciences for the FTIR microscopy, and Dr. Oduwole of Queen Mary and Westfield College for the ESR.

${ }^{1}$ L. T. Canham, Appl. Phys. Lett. 57, 1046 (1990).

${ }^{2}$ J. C. Vial, S. Billat, A. Bsiesy, G. Fishman, F. Gaspard, R. Herino, M. Ligeon, F. Madeore, I. Mihalcescu, F. Muller, and R. Romestain, Physica B 185, 593 (1993).

${ }^{3}$ M. Lannoo, C. Delerue, G. Allan, and E. Martin, Mater. Res. Soc. Symp. Proc. 358, 13 (1995).

${ }^{4}$ B. Delley and E. F. Steigmeier, Appl. Phys. Lett. 67, 2370 (1995).

${ }^{5}$ P. M. Derlet, T. C. Choy, and A. M. Stoneham, J. Phys. Condens. Matter 7, 2507 (1995).

${ }^{6}$ J. Penczek, A. Knoesen, H. W. H. Lee, and R. L. Smith, Mater. Res. Soc. Symp. Proc. 358, 641 (1995).

${ }^{7}$ A. Loni, A. J. Simons, T. I. Cox, P. D. J. Calcott, and L. T. Canham, Electron. Lett. 31, 1288 (1995).

${ }^{8}$ W. Lang, P. Steiner, F. Kozlowski, and H. Sandmeier, J. Lumin. 57, 169 (1993).

${ }^{9}$ T. Ito and A. Hiraki, J. Lumin. 57, 331 (1993).

${ }^{10}$ I. M. Chang, S. C. Pan, and Y. F. Chen. Phys. Rev. B 48, 8747 (1993).

${ }^{11}$ D. J. DiMaria, J. R. Kirtley, E. J. Pakulis, D. W. Dong, T. S. Kuan, F. L. Pesavento, T. N. Theis, J. A. Cutro, and S. D. Brorson, J. Appl. Phys. 56, 401 (1984).

${ }^{12}$ T. Komoda, J. P. Kelly, A. Nejim, K. P. Homewood, P. L. F. Hemment, and B. J. Sealy, Mater. Res. Soc. Symp. Proc. 358, 163 (1995).

${ }^{13}$ T. Shimizu-Iwayama, K. Fujita, S. Nakao, K. Saitoh, T. Fujita, and N. Itoh, J. Appl. Phys. 75, 7779 (1994).

${ }^{14}$ T. Shimizu-Iwayama, K. Fujita, S. Nakao, and N. Itoh, J. Phys. Condens. Matter 6, L601 (1994).

${ }^{15}$ Y. Ishikawa and N. Shibata, Nucl. Instrum. Methods 91, 520 (1994).

${ }^{16}$ Y. Ono, M. Nagase, M. Tabe, and Y. Takahashi, Jpn. J. Appl. Phys. 34, 1728 (1995).

${ }^{17}$ T. Fischer, T. Muschik, R. Schwarz, D. Kovalev, and F. Koch, Mater. Res. Soc. Symp. Proc. 358, 851 (1995).

${ }^{18}$ G. S. Tompa, D. C. Morton, B. S. Sywe, Y. Lu, E. W. Forsythe, J. A. Ott, D. Smith, J. Khugin, and B. A. Khan, Mater. Res. Soc. Symp. Proc. 358, 701 (1995).

${ }^{19}$ J. B. Khurgin, E. W. Forsythe, S. I. Kim, B. S. Sywe, B. A. Khan, and G. S. Tompa, Mater. Res. Soc. Symp. Proc. 358, 193 (1995).

${ }^{20}$ E. W. Forsythe, E. A. Whittaker, F. H. Pollak, B. S. Sywe, G. S. Tompa, B. A. Khan, J. Khurgin, H. W. H. Lee, F. Adar, and H. Schaffer, Mater. Res. Soc. Symp. Proc. 358, 187 (1995).

${ }^{21}$ S. Hayashi, T. Nagareda, Y. Kanzawa, and K. Yamamoto, Jpn. J. Appl. Phys. 32, 3840 (1993).

${ }^{22}$ S. Nozaki, H. Nakamura, H. Ono, H. Morisaki, and N. Ito, Jpn. J. Appl. Phys. 34, 122 (1994).

${ }^{23}$ L. T. Canham, Physica Status Solidi B 190, 9 (1995).

${ }^{24}$ B. H. Augustine, E. A. Irene, Y. J. He, K. J. Price, L. E. McNeil, K. N. Christensen, and D. M. Maher J. Appl. Phys. 78, 4020 (1995).

${ }^{25}$ Y. Kanemitsu, T. Ogawa, K. Shiraishi, and K. Takeda, Phys. Rev. B 48, 4883 (1993)

${ }^{26}$ M. Stutzmann, M. S. Brandt, M. Rosenbauer, H. D. Fuchs, S. Finkbeiner, J. Weber, and P. Deak, J. Lumin. 57, 321 (1993).

${ }^{27}$ J. G. Zhou, C. W. White, J. D. Budai, S. P. Withrow, and Y. Chen. Mater. Res. Soc. Symp. Proc. 358, 175 (1995). 
${ }^{28}$ T. Ito, K. Furuta, T. Yoneda, O. Arakaki, A. Hatta, and A. Hiraki, Mater. Res. Soc. Symp. Proc. 358, 477 (1995).

${ }^{29}$ V. Petrova-Koch, T. Muschik, G. Polisski, and D. Kovalev, Mater. Res. Soc. Symp. Proc. 358, 483 (1995).

${ }^{30}$ E. H. Poindexter, Z. Naturforsch. 50, 653 (1995).

${ }^{31}$ D. L. Griscom, J. Elect. Mater. 21, 763 (1992).

${ }^{32}$ N. A. Hill and K. B. Whaley, Mater. Res. Soc. Symp. Proc. 358, 477 (1995).

${ }^{33}$ L. Brus, IEEE J. Quantum Electron. QE-22, 1909 (1986).
${ }^{34}$ M. A. Stevens Kalceff and M. R. Phillips, Phys. Rev. B 52, 3122 (1995).

${ }^{35}$ S. Munekuni, T. Yamanaka, Y. Shimogaichi, R. Tohmon, Y. Ohki, K. Nagaswawa, and Y. Hama, J. Appl. Phys. 68, 1212 (1990).

${ }^{36}$ H. Koyama, J. Appl. Phys. 51, 2228 (1980).

${ }^{37}$ M. Lannoo, C. Delerue, and G. Allan, J. Lumin. 57, 243 (1993).

${ }^{38}$ C. Delerue, M. Lannoo, G. Allan, and E. Martin, Thin Solid Films 255, 27 (1995).

${ }^{39}$ M. Hirao and T. Uda, Int. J. Quantum Chem. 52, 1113 (1995). 